Modular representation theory of blocks with trivial intersection defect groups

An, Jainbei and Eaton, Charles W.

2005

MIMS EPrint: 2009.69

Manchester Institute for Mathematical Sciences

School of Mathematics

The University of Manchester

\footnotetext{
Reports available from: http://eprints.maths.manchester.ac.uk/

And by contacting: The MIMS Secretary

School of Mathematics

The University of Manchester

Manchester, M13 9PL, UK
} 


\title{
Modular Representation Theory of Blocks with Trivial Intersection Defect Groups
}

\author{
JIANBEI AN ${ }^{1}$ and CHARLES W. EATON ${ }^{2, \star \star ~}$ \\ ${ }^{1}$ Department of Mathematics, University of Auckland, Auckland, New Zealand. \\ e-mail:an@math.auckland.ac.nz \\ ${ }^{2}$ School of Mathematics, University of Birmingham, Birmingham B15 2TT, U.K. \\ e-mail:eatonc@for.mat.bham.ac.uk
}

(Received: September 2003; accepted: January 2004)

Presented by A. Verschoren

Abstract. We show that Uno's refinement of the projective conjecture of Dade holds for every block whose defect groups intersect trivially modulo the maximal normal $p$-subgroup. This corresponds to the block having $p$-local rank one as defined by Jianbei An and Eaton. An immediate consequence is that Dade's projective conjecture, Robinson's conjecture, Alperin's weight conjecture, the IsaacsNavarro conjecture, the Alperin-McKay conjecture and Puig's nilpotent block conjecture hold for all trivial intersection blocks.

Mathematics Subject Classification (2000): Primary 20C20.

Key words: modular representations, Dade's projective conjecture, $p$-local rank, blocks, cyclic defect groups.

\section{Introduction}

In this paper we verify that the refinement by Uno of Dade's projective conjecture (DPC) generalising the Isaacs-Navarro conjecture holds whenever a block $B$ of a finite group $G$ has defect groups which intersect pairwise in $O_{p}(G)$ (blocks of $p$ local rank one as defined in [2]). DPC is already known to be true when additionally $B$ has defect groups which are Sylow $p$-subgroups (see [11], extending results of [7]), the proof depending on the classification detailed in [17] and [30]. We make use of the classification of the blocks with trivial intersection defect groups given in [4].

We briefly review the notation necessary to state the conjecture. Let $G$ be a finite group and $p$ a prime. We consider $p$-chains of $p$-subgroups $\sigma: Q_{0}<\cdots<Q_{n}$ (where $<$ denotes strict inclusion), and write $|\sigma|=n$ for the length of $\sigma$. The

$\star$ This research was supported in part by the Marsden Fund of New Zealand via grant \#9144/3368248.

$\star \star$ Current address: School of Mathematics, University of Manchester, Sackville Street, PO Box 88, Manchester M60 1QC, U.K. e-mail: charles.eaton@manchester.ac.uk 
stabilizer under the natural conjugation action of $G$ is $G_{\sigma}=N_{G}(\sigma)=N_{G}\left(Q_{0}\right) \cap$ $\cdots \cap N_{G}\left(Q_{n}\right)$. Write $\sigma_{i}$ for the initial subchain $Q_{0}<\cdots<Q_{i}$. Denote by $\mathcal{C}(G)$ the set of all such chains and if $\mathcal{X}(G)$ is some subset of $\mathcal{C}(G)$, we denote by $\mathcal{X}(G \mid Q)$ the set of chains in $\mathcal{X}(G)$ with initial subgroup $Q$ and by $\mathcal{X}(G) / A$ a set of orbit representatives under the action of a group of automorphisms $A$ provided that $\mathcal{X}(G)$ is an $A$-set. A $p$-subgroup $Q$ of $G$ is radical if $Q=O_{p}\left(N_{G}(Q)\right)$, and the $p$-chain $\sigma$ is radical if $Q_{i}=O_{p}\left(N_{G}\left(\sigma_{i}\right)\right)$ for each $i$. Denote by $\mathcal{R}(G)$ the set of radical $p$-chains of $G$.

Let $\mathcal{O}$ be a local complete discrete valuation ring containing a primitive $|G|^{3}$ root of unity, whose residue field $k=\mathcal{O} / J(\mathcal{O})$ is algebraically closed of characteristic $p$ and whose field of fractions $K$ has characteristic zero. Denote by $\operatorname{Blk}(G)$ the set of blocks of $G$ with respect to this system and let $B \in \operatorname{Blk}(G)$. If $H \leqslant G$, then denote by $\operatorname{Blk}(H, B)$ the set of blocks of $H$ with Brauer correspondent $B$.

Following [2] and [27], define the $p$-local rank to be $\operatorname{plr}(B)=\max \{|\sigma|$ : $\sigma \in \mathcal{R}(G, B)\}$, where $\mathcal{R}(G, B) \subseteq \mathcal{R}(G)$ consists of those chains $\sigma$ for which $\operatorname{Blk}\left(G_{\sigma}, B\right) \neq \emptyset$. Define the $p$-local rank of $G$ to be $\operatorname{plr}\left(B_{0}\right)$, where $B_{0}=B_{0}(G)$ is the principal $p$-block of $G$. By $[2,5.1] \operatorname{prr}(B)=1$ if and only if $B$ has a defect group $D$ for which $D / O_{p}(G) \neq 1$ and is a trivial intersection (TI) subgroup of $G / O_{p}(G)$ (recall that a subgroup $H$ of $G$ is a trivial intersection subgroup of $G$ if $H^{g} \cap H=1$ whenever $\left.g \in G \backslash N_{G}(H)\right)$. It follows in this case that $D$ contains no radical $p$-subgroups aside from $O_{p}(G)$ and itself, so that $\mathcal{R}(G, B) / G$ may be taken to be $\left\{O_{p}(G), D, O_{p}(G)<D\right\}$. Following [3], when $\operatorname{plr}(B)=1$ and $O_{p}(G)=1$, we refer to $B$ as a TI defect block.

Write $\operatorname{Irr}(G)$ for the set of irreducible characters of $G$, and $\operatorname{Irr}_{d}(G)$ for the set of those $\chi \in \operatorname{Irr}(G)$ with defect $d$ (i.e., $p^{d} \chi(1)_{p}=|G|_{p}$ ). If $N \unlhd G$ and $\mu \in \operatorname{Irr}(N)$, then write $\operatorname{Irr}(G, \mu)$ for the set of irreducible characters of $G$ covering $\mu$. Denote by $\operatorname{Irr}(G, B)$ the set of irreducible characters belonging to $B$, and more generally, for $H \leqslant G$ write $\operatorname{Irr}(H, B)$ for the set of irreducible characters of $H$ belonging to Brauer correspondents of $B$. For brevity we sometimes write, for example, $\operatorname{Irr}(B)$ for $\operatorname{Irr}(G, B)$ when $B$ is a block of $G$. Write $k(G), k_{d}(G)$, etc. for the cardinality of these sets. Write $l(H, B)$ for the number of isomorphism classes of simple $k H$ modules belonging to Brauer correspondents of $B$, and write $l_{B}(Q)$ for the number of isomorphism classes of simple $k G$-modules in $B$ with vertex $Q$. Following [27] and [9], for a normal $p$-subgroup $Q$ of $G$ write $w(G, Q)$ for the number of $Q$ projective irreducible characters of $G$, i.e., those characters $\chi \in \operatorname{Irr}(G)$ satisfying $\chi(1)_{p}=[G: Q]_{p} \mu(1)$ whenever $\mu \in \operatorname{Irr}(Q)$ is covered by $\chi$ (or alternatively, $\chi$ may be afforded by a $Q$-projective $\mathcal{O} G$-module). We combine all of the above notations freely.

Dade's projective conjecture (in its most general form) is as follows: 
CONJECTURE 1.1 (Dade [9]). Let $G$ be a finite group and $B \in \operatorname{Blk}(G)$. Then for every $\lambda \in \operatorname{Irr}\left(O_{p}(Z(G))\right)$ and every $d$ we have

$\sum_{\sigma \in \mathcal{R}\left(G \mid O_{p}(G)\right) / G}(-1)^{|\sigma|} k_{d}\left(G_{\sigma}, B, \lambda\right)=\sum_{\sigma \in \mathcal{R}\left(G \mid O_{p}(G)\right) / G}(-1)^{|\sigma|} w_{d}\left(G_{\sigma}, B, \lambda, O_{p}(G)\right)$.

Remarks. (i) This is of course trivially true when $O_{p}(G)$ is a defect group for $B$, so we assume otherwise. If $O_{p}(G) \leqslant Z(G)$ (and the defect groups of $G$ strictly contain $O_{p}(G)$ ), then the right-hand-side of the equation predicted by the equation is zero. If further $\operatorname{plr}(B)=1$, then the conjecture predicts that $k_{d}(G, B, \lambda)=$ $k_{d}\left(N_{G}(D), B, \lambda\right)$ whenever $D$ is a defect group for $B$.

(ii) When $\operatorname{prr}(B)=1$, the above conjecture is identical to Robinson's conjecture $[27,4.1]$.

Let $H \leqslant G, \varphi \in \operatorname{Irr}(H)$ and let $r(\varphi)=r_{p}(\varphi)$ be the integer $0<r(\varphi) \leqslant(p-1)$ such that the $p^{\prime}$-part $(|H| / \varphi(1))_{p^{\prime}}$ of $|H| / \varphi(1)$ satisfies

$$
\left(\frac{|H|}{\varphi(1)}\right)_{p^{\prime}} \equiv r(\varphi) \quad(\bmod p)
$$

Given an integer $1 \leqslant r \leqslant(p-1) / 2$, let $\operatorname{Irr}(H,[r])$ be the subset of $\operatorname{Irr}(H)$ consisting of those characters $\varphi$ such that $r(\varphi) \equiv \pm r(\bmod p)$, and let

$$
\operatorname{Irr}_{d}(H, B, \lambda,[r])=\operatorname{Irr}_{d}(H, B, \lambda) \cap \operatorname{Irr}(H,[r])
$$

and $k_{d}(H, B, \lambda,[r])=\left|\operatorname{Irr}_{d}(H, B, \lambda,[r])\right|$.

Let $B \in \operatorname{Blk}(G)$ with defect group $D=D(B)$ and Brauer correspondent $b \in$ $\operatorname{Blk}\left(N_{G}(D)\right)$. Then

$$
k_{d(B)}\left(G_{\sigma}, B,[r]\right)=\sum_{\lambda \in \operatorname{Irr}\left(O_{p}(Z(G))\right)} k_{d(B)}\left(G_{\sigma}, B, \lambda,[r]\right)
$$

is the number of characters $\varphi \in \operatorname{Irr}(b)$ such that $\varphi$ has height 0 and $r(\varphi) \equiv$ $\pm r(\bmod p)$, where $d(B)$ is the defect of $B$. The following is the Isaacs-Navarro conjecture.

CONJECTURE 1.2 (Conjecture B [20]). In the notation above,

$$
k_{d(B)}(G, B,[r])=k_{d(B)}\left(N_{G}(D), B,[r]\right) .
$$

The Isaacs-Navarro conjecture is a refinement of the Alperin-McKay conjecture. The following refinement of Dade's projective conjecture is due to Uno. We state it in a more general (but equivalent - see [12]) form. In its original form it is assumed that $O_{p}(G) \leqslant Z(G)$ and that $O_{p}(G)$ is not a defect group for $B$. 
CONJECTURE 1.3 (Conjecture 3.2 [32]). Let $G$ be a finite group and $B \in \operatorname{Blk}(G)$. Then, for every $\lambda \in \operatorname{Irr}\left(O_{p}(Z(G))\right)$, every $d$ and every $r$ with $1 \leqslant r \leqslant(p-1) / 2$, we have

$$
\begin{aligned}
& \sum_{\sigma \in \mathcal{R}\left(G \mid O_{p}(G)\right) / G}(-1)^{|\sigma|} k_{d}\left(G_{\sigma}, B, \lambda,[r]\right) \\
= & \sum_{\sigma \in \mathcal{R}\left(G \mid O_{p}(G)\right) / G}(-1)^{|\sigma|} w_{d}\left(G_{\sigma}, B, \lambda,[r], O_{p}(G)\right) .
\end{aligned}
$$

Remarks. (i) If $p=2$ or 3 , then the conjecture is equivalent to Dade's projective conjecture.

(ii) It is clear that this is a direct refinement of Conjecture 1.1. The proof that it is a strengthening of the Isaacs-Navarro conjecture is not available at the time of writing. However, when $\operatorname{prr}(B)=1$ the Isaacs-Navarro conjecture is in fact a special case (since the terms on the right hand side are all zero when $d$ is the defect of the block (except when $O_{p}(G)$ is a defect group for $B$ ) and the only radical chains used are of the form $O_{p}(G)$ or $O_{p}(G)<D$, where $D$ is a defect group of $B$ ). For this reason we treat only Uno's version explicitly, remarking that Isaacs-Navarro's conjecture is a consequence.

The main result of this paper is as follows:

THEOREM 1.4. Let $G$ be a finite group and $B$ a p-block of $G$. If $\operatorname{plr}(B) \leqslant 1$, then Conjecture 1.3 holds for $B$. In particular, $k_{d}(G, B,[r])=k_{d}\left(N_{G}(D), B,[r]\right)$ for any integers $d$ and $r$, when $O_{p}(G) \leqslant Z(G)$.

We give some consequences.

COROLLARY 1.5. Both the Isaacs-Navarro conjecture (and so the AlperinMcKay conjecture) and Alperin's weight conjecture hold for every block satisfying the conditions of Theorem 1.4. In particular, $l(G, B)=l_{B}\left(O_{p}(G)\right)+l\left(N_{G}(D), B\right)$.

Proof. We have already discussed the Isaacs-Navarro and Alperin-McKay conjectures, so consider Alperin's weight conjecture.

First assume that $O_{p}(G)=1$. Theorem 1.4 tells us that $k(G, B)=k\left(N_{G}(D), B\right)$ (summing over all integers $d, r$ ), and so $l(G, B)=l\left(N_{G}(D), B\right.$ ) (by the results of [21], after cancelling nonradical chains). But this is precisely the statement of Alperin's weight conjecture for a block with TI defect groups (since there are no non-trivial radical $p$-subgroups contained in $D$ ). Now suppose $O_{p}(G) \neq 1$, write $\bar{G}=G / O_{p}(G)$. It is easy to see that if Alperin's conjecture holds for every block $\bar{B}$ of $\bar{G}$ contained in $B$, then it holds for $B$, so the result follows by the previous argument.

COROLLARY 1.6. Conjecture 1.3 and Alperin's weight conjecture hold whenever B is a TI block. 
Proof. This follows immediately from Theorem 1.4 and Corollary 1.5 since every TI block has TI defect groups by $[3,3.3]$.

Watanabe in [33] shows that if Alperin's weight conjecture holds, then a question of Puig's (see [26, 1.9]) relating to nilpotent blocks has a positive answer. We apply Corollary 1.5 to give a special case.

COROLLARY 1.7. Let $B \in \operatorname{Blk}(G)$ have p-local rank at most one. Then $B$ is nilpotent if and only if $l\left(Q C_{G}(Q), b_{Q}\right)=1$ for every $B$-subgroup $\left(Q, b_{Q}\right)$.

Proof. We must show that if Alperin's weight conjecture holds for blocks of $p$ local rank at most one, then any such block $B$ is nilpotent if and only if $l\left(Q C_{G}(Q)\right.$, $\left.b_{Q}\right)=1$ for every $B$-subgroup $\left(Q, b_{Q}\right)$. The argument given in [33] uses induction on $|G|$, so cannot be used directly. However we observe that in the proof of Proposition 5 of [33], induction on $|G|$ is invoked only twice (this occurring in the first paragraph). The blocks involved in the inductive steps, $b_{Q}$ and $\bar{B}$ in the notation of [33], each have $p$-local rank at most one by Proposition 2.1 (ii) and (iii) respectively, and hence we may indeed apply the argument of [33].

\section{The $p$-local Rank}

We include for the convenience of the reader some properties of the $p$-local rank of a block and of TI defect blocks. Proofs may be found in [3] and [4].

PROPOSITION 2.1. Let $B \in \operatorname{Blk}(G)$ have defect group D. Let $H \leqslant G$ and $N \unlhd G$. Then:

(i) $\operatorname{prr}(B)=0$ if and only if $D \unlhd G ; \operatorname{prr}(B) \leqslant 1$ if and only if $D / O_{p}(G)$ is TI;

(ii) if $b \in \operatorname{Blk}(H, B)$, then $\operatorname{prr}(b) \leqslant \operatorname{plr}(G)$;

(iii) suppose $\mu \in \operatorname{Irr}(N)$ is $G$-stable and extends to $\theta \in \operatorname{Irr}(G)$; if $\psi \in \operatorname{Irr}(G / N)$ lies in the block $b_{N}$ of $G / N$ and $\theta \psi \in \operatorname{Irr}(G, B)$, then $\operatorname{prr}\left(b_{N}\right) \leqslant \operatorname{plr}(B)$; if further $\operatorname{pr}(B)=1$ and $O_{p}(G)=1$, then $b_{N}$ either has a normal defect group or TI defect groups;

(iv) if $G=G_{1} \times G_{2}$ and $B=B_{1} \times B_{2}$, where $B_{i} \in \operatorname{Blk}\left(G_{i}\right)$, then $\operatorname{plr}(B)=$ $\operatorname{plr}\left(B_{1}\right)+\operatorname{plr}\left(B_{2}\right)$;

(v) if $b \in \operatorname{Blk}(N)$ is covered by $B$, with $\operatorname{prr}(B)=1$ and $O_{p}(G)=1$, then $\operatorname{plr}(b)=0$ or $\operatorname{plr}(b)=1$ and $O_{p}(N)=1$;

(vi) if $N \leqslant Z(G), \operatorname{plr}(B)=1$ and $\bar{B}$ is the unique block of $G / N$ corresponding to $B$ under the natural epimorphism, then $\operatorname{pr}(\bar{B})=1$.

\section{Character Correspondences}

Here we summarize the character correspondences needed for the reduction step. We use the methods of [4], and apply the work of Külshammer and Puig in [24] 
to move from covered blocks of defect zero to covered blocks with central defect group.

Let $B \in \operatorname{Blk}(G), Z=O_{p}(Z(G))$ and $N \unlhd G$. Suppose that $B$ covers $b \in$ $\operatorname{Blk}(N)$, where $b$ is $G$-stable and has defect group $D_{0}$ contained in $Z$. Let $D$ be a defect group of $B$, so $D \cap N=D_{0}$.

As a block with central defect group $D_{0}$, we have $k(N, b, \lambda)=1$ for each $\lambda \in \operatorname{Irr}\left(D_{0}\right)$. Fix $\lambda_{0} \in \operatorname{Irr}\left(D_{0}\right)$, and let $\operatorname{Irr}\left(N, b, \lambda_{0}\right)=\{\zeta\}$, so $I_{G}(\zeta)=G$. We may choose a central extension $\hat{G}$ of $G$ (with $\hat{W} \leqslant Z(\hat{G})$ where $\hat{G} / \hat{W} \cong G$ ) such that there is an irreducible character $\hat{\theta} \in \operatorname{Irr}(\hat{G})$ extending $\zeta$. Let $\hat{N}$ be the subgroup of $\hat{G}$ identified with $N$, so that $\hat{N} \cap \hat{W}=1$. Set $\tilde{G}=\hat{G} / \hat{N}$, a central extension of $G / N$ by $\tilde{W}$ (where $\tilde{W}$ is the image of $\hat{W}$ under the natural homomorphism). Thus $\hat{\theta}$ lies over a unique linear character $\hat{\mu}$ of $\hat{W}$. Let $\tilde{\mu}$ be the complex conjugate of $\hat{\mu}$, regarded as a character of $\tilde{W}$. There is a one-to-one correspondence between $\operatorname{Irr}(G, \zeta)$ and $\operatorname{Irr}(\tilde{G}, \tilde{\mu})$, given by $\chi \leftrightarrow \hat{\theta} \tilde{\chi}$, where, of course, we are identifying $\chi$ with its inflation to $\hat{G}$. Now there is a collection of blocks $\tilde{B}_{1}, \ldots, \tilde{B}_{r}$ of $\tilde{G}$ (the Dade correspondents of $B$ ) so that, writing $\tilde{B}=\tilde{B}_{1}+\cdots+\tilde{B}_{r}$, there is a correspondence between $\operatorname{Irr}(G, B, \zeta)$ and $\operatorname{Irr}(\tilde{G}, \tilde{B}, \tilde{\mu})$, where implicitly we are using the one-to-one correspondence between blocks of $G$ and blocks of $\hat{G}$ given by the natural epimorphism. Let $\hat{B}$ be the unique block of $\hat{G}$ corresponding to $B$. The Dade correspondence respects the Brauer correspondence as described in [13]. By the results of [24] we have $r=1$.

\section{Reductions}

In this section we give the reductions which state that in order to prove Theorem 1.4 it suffices to consider the situation where $F(G) \leqslant Z(G)$ and $F^{*}(G) / Z(G)$ is non-abelian simple (i.e., $G$ is a covering group of a group $Y$ of the form $X \leqslant Y \leqslant$ $\operatorname{Aut}(X)$ where $X$ is non-Abelian simple). Throughout this section we consider the following hypotheses.

HYPOTHESES 4.1. B is a block of p-local rank one of $G$ for which Conjecture 1.3 fails, and first $[G: Z(G)]$, then $|G|$ are minimized subject to the presence of such a block. Let $D$ be a defect group for $B$.

Although the reductions can be achieved through the application of the results of [28] and [29], this can only be achieved through detailed examination of the proofs, ensuring that each step is compatible with the $p$-local rank and also with respect to the refinement involved in Uno's conjecture. In the interests of completeness, we provide reductions here (except where reductions exist which make explicit reference to the $p$-local rank). The ideas are broadly similar to those used in [29], but are a great deal simpler due to our specific situation.

PROPOSITION 4.2. Under Hypotheses $4.1 O_{p}(G) \leqslant Z(G) \cap G^{\prime}$.

Proof. This is $[12,1.8]$. 
PROPOSITION 4.3. Consider Hypotheses 4.1. Suppose that $N \unlhd G$ and that $B$ covers $b \in \operatorname{Blk}(N)$. Then $b$ is $G$-stable, i.e., $G=I_{G}(b)$.

Proof. By $[1,15.1]$ we may choose defect groups $D_{0}$ of $b$ and $D$ of $B$ such that $D_{0}=D \cap N$ and $D \leqslant I=I_{G}(b)$. Let $B_{I}$ be the unique block of $I$ covering $b$ with Brauer correspondent $B$. Necessarily, $B_{I}$ and $B$ have a defect group in common, and we may take this to be $D$ (see [14, V.2.5]). Let $\lambda \in \operatorname{Irr}(Z)$, where $Z=O_{p}(Z(G))$. Let $d$ be an integer and $r \in\{1, \ldots, p-1\}$. Now $I_{G}(\zeta) \leqslant I$ for each $\zeta \in \operatorname{Irr}(N, b)$, so

$k_{d}(G, B, \lambda,[r])=\sum_{\zeta \in \operatorname{Irr}(N, b) / G} k_{d}(G, B, \lambda, \zeta,[r])=\sum_{\zeta \in \operatorname{Irr}(N, b) / G} k_{d}\left(I, B_{I}, \lambda, \zeta,[r]\right)$

by Clifford theory (we are also using Frobenius reciprocity to ensure that characters lie over $\lambda$ where necessary). Observe that $I$ controls fusion in $\operatorname{Irr}(N, b)$, so this is

$$
\sum_{\zeta \in \operatorname{Irr}(N, b) / I} k_{d}\left(I, B_{I}, \lambda, \zeta,[r]\right)=k_{d}\left(I, B_{I}, \lambda,[r]\right) .
$$

We move to the Brauer correspondent for $b$ in $N_{N}\left(D_{0}\right)$. However, as will become apparent, it is necessary to consider instead of $b$, the unique block $\tilde{b}$ of $D N$ covering $b$ (such a block of $D N$ exists by $[14$, V.3.5]). Since $D \leqslant I$, by $[1,15.1]$ $D$ is a defect group for $\tilde{b}$. Let $\tilde{b}_{1}$ be the unique Brauer correspondent for $\tilde{b}$ in $N_{D N}(D)=D N_{N}(D)$. Note that $N_{D N}(D) \unlhd N_{G}(D)$.

Let $x \in I_{N_{G}(D)}\left(\tilde{b}_{1}\right)$. Then $\tilde{b}^{x}=\left(\tilde{b}_{1}^{D N}\right)^{x}=\left(\tilde{b}_{1}^{x}\right)^{x^{-1} D N x}=\left(\tilde{b}_{1}\right)^{D N}=\tilde{b}$. So $x \in I$. Hence $I_{N_{G}(D)}\left(\tilde{b}_{1}\right) \leqslant N_{I}(D)$. Hence the Brauer correspondence gives a oneto-one correspondence between blocks of $N_{G}(D)$ covering $\tilde{b}_{1}$ and blocks of $N_{I}(D)$ covering $\tilde{b}_{1}$.

Let $B_{I}^{\prime}$ be the Brauer correspondent of $B_{I}$ in $N_{I}(D)$, and let $B^{\prime}$ be the Brauer correspondent of $B$ in $N_{G}(D)$. By [18] $B_{I}^{\prime}$ covers $\tilde{b}_{1}$, as does $B^{\prime}$. Hence the transitivity of the Brauer correspondence and a repetition of the above argument ensures that

$$
\begin{aligned}
k_{d} & \left(N_{G}(D), B, \lambda,[r]\right) \\
& =\sum_{\zeta \in \operatorname{Irr}\left(N_{D N}(D), \tilde{b}\right) / N_{G}(D)} k_{d}\left(N_{G}(D), B, \lambda, \zeta,[r]\right) \\
& =\sum_{\zeta \in \operatorname{Irr}\left(N_{D N}(D), \tilde{b}\right) / N_{I}(D)} k_{d}\left(N_{I}(D), B_{I}, \lambda, \zeta,[r]\right)=k_{d}\left(N_{I}(D), B_{I}, \lambda,[r]\right) .
\end{aligned}
$$

Now since $B_{I}^{G}=B$, we have $\operatorname{pr}\left(B_{I}\right) \leqslant \operatorname{pr}(B)=1$. Hence, if $I \neq G$, then

$$
\begin{aligned}
k_{d}(G, B, \lambda,[r]) & =k_{d}\left(I, B_{I}, \lambda,[r]\right)=k_{d}\left(N_{I}(D), B_{I}, \lambda,[r]\right) \\
& =k_{d}\left(N_{G}(D), B, \lambda,[r]\right),
\end{aligned}
$$

either because $Z<O_{p}(I)$ and this equality holds for trivial reasons, or $Z=O_{p}(I)$ and Conjecture 1.3 holds for $B_{I}$ by induction. Hence, Dade's conjecture does hold for $B$, contradicting our choice of $B$ as a counterexample. 
PROPOSITION 4.4. Under the hypotheses of Proposition 4.3, if $b$ has a defect group central in $G$, then $N \leqslant Z(G)$.

Proof. In this case it is possible to apply the results of [24] as in [29]. However, in our special situation it is possible to use more elementary methods based on the contents of Section 3. Our arguments here are broadly a simplification of those in [29].

Suppose that $b$ has defect group $D_{0} \leqslant Z=O_{p}(Z(G))$. Let $\zeta \in \operatorname{Irr}(N, b)$. By Proposition 4.3 we have $I_{G}(\zeta)=G$. Let $D$ be a defect group of $B$, so that $D \cap N=$ $D_{0}$. Take the notation of Section 3 , so that $\tilde{B}$ is the unique Dade correspondent of $B$ (with respect to $\zeta$ ).

Now let $H=N N_{G}(D)$. Then $N_{H}(D)=N_{G}(D)$. Let $A$ be the unique Brauer correspondent of $B$ for $N_{G}(D)$. The Brauer correspondent $A^{H}$ of $A$ for $H$ exists, satisfies $\left(A^{H}\right)^{G}=B$ and has $D$ as a defect group. Note that $C=A^{H}$ also covers $b$. Since $\zeta \in \operatorname{Irr}(N, b)$ extends to $\hat{G}$, it also extends to $\hat{H} \leqslant \hat{G}$, a central extension of $H$ by $\hat{W}$. Hence we may apply the whole of Section 3 to $H$ and $C$, obtaining an unique Dade correspondent $\tilde{C}$, a block of $\tilde{H}$, etc. Let $\lambda \in \operatorname{Irr}(Z)$. Suppose $\lambda$ covers $\lambda_{0} \in \operatorname{Irr}\left(D_{0}\right)$. Then $\lambda$ may be written uniquely as $\lambda_{0} \lambda_{1}$, where $\lambda_{1}$ is the inflation of an irreducible character of $Z / D_{0}$ (once we have chosen an extension of $\lambda_{0}$ to $Z$ ). Now $D N \unlhd H$, and $D N / N \unlhd H / N$. Write $\tilde{D} \leqslant \tilde{H}$ for the relevant central extension of $D N / N$. Now $\tilde{C}$ has $\tilde{D}$ as a defect group, and by the character correspondences given in Section 3 we have

$$
k_{d}(G, B, \lambda,[r])=k_{\tilde{d}}\left(\tilde{G}, \tilde{B}, \tilde{\lambda}_{0} \tilde{\lambda}_{1},[\tilde{r}]\right)
$$

and

$$
k_{d}\left(N N_{G}(D), C, \lambda,[r]\right)=k_{\tilde{d}}\left(\tilde{H}, \tilde{C}, \tilde{\lambda}_{0} \tilde{\lambda}_{1},[\tilde{r}]\right)
$$

for each integer $d$. Here

$$
\tilde{d}=d+\log _{p}\left(\zeta(1)_{p}|\tilde{W}|_{p} /|N|_{p}\right)=d+\log _{p}\left(|\tilde{W}|_{p} /\left|D_{0}\right|_{p}\right),
$$

$\tilde{\lambda}_{1}$ is the inflation of $\lambda_{1}$ to the subgroup of $\tilde{G}$ corresponding to $Z$, and $\tilde{r} \equiv$ $\left(|\tilde{W}|_{p^{\prime}} \zeta(1)_{p^{\prime}}\right) /\left(|N|_{p^{\prime}}\right) r(\bmod p) . \operatorname{But} \operatorname{plr}(\tilde{B})=1$ with $O_{p}(\tilde{G})=O_{p}(\tilde{W}) \leqslant Z(\tilde{G})$ and defect group $\tilde{D}, \tilde{H}=N_{\tilde{G}}(\tilde{D})$, and $[\tilde{G}: Z(\tilde{G})]<[G: Z(G)]$, so the conjecture holds for $\tilde{B}$, and

$$
k_{\tilde{d}}\left(\tilde{G}, \tilde{B}, \tilde{\lambda}_{0} \tilde{\lambda}_{1},[\tilde{r}]\right)=k_{\tilde{d}}\left(\tilde{H}, \tilde{C}, \tilde{\lambda}_{0} \tilde{\lambda}_{1},[\tilde{r}]\right) .
$$

It remains to show that $k_{d}\left(N N_{G}(D), C, \lambda,[r]\right)=k_{d}\left(N_{G}(D), B, \lambda,[r]\right)$, so that $k_{d}\left(N_{G}(D), B, \lambda,[r]\right)=k_{d}(G, B, \lambda,[r])$ and $B$ is not a counterexample to the conjecture after all. If $H=N N_{G}(D)=N_{G}(D)$, then we are done, so suppose otherwise. Recall that $N_{H}(D)=N_{G}(D)$ and that $D$ is a defect group for $C$. Since we are assuming $N_{H}(D) \neq H, H$ must possess a distinct conjugate $D^{h}$ of $D$. Then $O_{p}(H) \leqslant D^{h} \cap D=Z$. Further $\operatorname{plr}(C)=1$ and $[H: Z(H)]<[G: Z(G)]$, so the conjecture holds for $C$, and $k_{d}(H, C, \lambda,[r])=k_{d}\left(N_{H}(D), C, \lambda,[r]\right)$ as required. 
COROLLARY 4.5. Consider Hypotheses 4.1. Then $O_{p^{\prime}}(G) \leqslant Z(G)$.

Proof. This is immediate since every block of $O_{p^{\prime}}(G)$ has defect zero.

Consider $E(G)$, the normal subgroup generated by the components of $G$ (recall that a component is a subnormal quasisimple subgroup). This is a central product $M_{1} * \cdots * M_{r}$ of normal subgroups of $G$, where each $M_{i}$ is a central product $M_{i 1} * \cdots * M_{i s}$ of quasisimple groups.

COROLLARY 4.6. Consider Hypotheses 4.1. Then $E(G)$ is quasisimple.

Proof. Let $b \in \operatorname{Blk}(E(G))$. Then $b=b_{1} \times \cdots \times b_{r}$ for $b_{i} \in \operatorname{Blk}\left(M_{i}\right)$. By Proposition 4.4, $b_{i}$ has noncentral defect groups (since it is covered by $B$ and since $Z\left(M_{i}\right) \leqslant Z(G)$ ). Hence $\operatorname{plr}\left(b_{i}\right)>0$. But by Proposition $2.11=$ $\operatorname{plr}(B) \geqslant \operatorname{plr}(b)=\operatorname{plr}\left(b_{1}\right)+\cdots+\operatorname{plr}\left(b_{r}\right) \geqslant r$. So $r=1$ and $\operatorname{plr}\left(b_{1}\right)=1$. Now $b_{1}=b_{11} \times \cdots \times b_{1 s}$, where $b_{1 i} \in \operatorname{Blk}\left(M_{1 i}\right)$. By Proposition $4.3 b_{1}$ is $G$ stable. Since $G$ permutes the $M_{1 i}$ transitively, this means that $b_{11} \cong b_{1 i}$ for each $i$. Hence, by Proposition $2.11=\operatorname{prr}\left(b_{1}\right)=s\left(\operatorname{plr}\left(b_{11}\right)\right)$, and $s=1$ as required.

PROPOSITION 4.7. Under Hypotheses 4.1, $M \leqslant G / Z(G) \leqslant \operatorname{Aut}(M)$ for some non-Abelian simple group $M$.

Proof. Immediate from the previous two corollaries.

It is worth noting here that we only need consider automorphisms which fix each element of the centre of the covering group of our simple group.

\section{Blocks with Cyclic Defect Groups}

Although the case where $D / O_{p}(G)$ is cyclic follows directly from the results of the next section and the proof of the Isaacs-Navarro conjecture for blocks with cyclic defect groups given in [20], we digress in this section in order to prove a stronger result in this direction. We first state Uno's refinement of Dade's invariant projective conjecture, then prove the conjecture for all blocks with cyclic defect groups. We state the refinement in a character version but prove a twisted group algebra version which is equivalent to the character one.

In the notation of Section 1 , let $E$ be an extension of $G, F=E / G, \sigma \in$ $\mathcal{R}(G), \psi \in \operatorname{Irr}\left(G_{\sigma}\right)$ and $N_{E}(\sigma, \psi)$ the stabilizer of $(\sigma, \psi)$. Then $N_{F}(\sigma, \psi)=$ $N_{E}(\sigma, \psi) / G_{\sigma} \leqslant F$. For $U \leqslant E$, denote $\operatorname{Irr}\left(G_{\sigma}, U\right)$ the subset of $\operatorname{Irr}\left(G_{\sigma}\right)$ consisting of characters $\psi$ such that $N_{F}(\sigma, \psi)=U$ and let

$$
\operatorname{Irr}_{d}\left(G_{\sigma}, B, U, \lambda\right)=\operatorname{Irr}_{d}\left(G_{\sigma}, B, \lambda\right) \cap \operatorname{Irr}\left(G_{\sigma}, U\right)
$$

and $k_{d}\left(G_{\sigma}, B, U, \lambda\right)=\left|\operatorname{Irr}_{d}\left(G_{\sigma}, B, U, \lambda\right)\right|$. The following is Uno's refinement of Dade's invariant projective conjecture. 
CONJECTURE 5.1 ([32]). Let $E$ be an extension of a finite group $G$ with $O_{p}(G) \leqslant$ $Z(G)$, and let $F=E / G, B \in \operatorname{Blk}(G)$ with $D(B)>O_{p}(G)$. Then for every $\lambda \in \operatorname{Irr}\left(O_{p}(Z(G))\right), U \leqslant F$, every $d$ and every $r$ with $1 \leqslant r \leqslant(p-1) / 2$, we have

$$
\sum_{\sigma \in \mathcal{R}\left(G \mid O_{p}(G)\right) / G}(-1)^{|\sigma|} k_{d}\left(G_{\sigma}, B, \lambda, U,[r]\right)=0 .
$$

THEOREM 5.2. Let $G$ be a finite group with $O_{p}(G) \leqslant Z(G)$, and let $B \in \operatorname{Blk}(G)$ such that $D(B) / O_{p}(G)$ is cyclic. Then Conjecture 5.1 holds for $B$.

Proof. We will prove a refinement of [10, Conjecture 7.9], which is equivalent to Conjecture 5.1 for the blocks with cyclic defect groups. We will use the notation of [10].

Case (1) The corresponding result of Uno's refinement to the ordinary theorem, [10, Theorem 4.3], holds, that is, in the notation of [10, Theorem 4.3] $\operatorname{Irr}(B,[r])$ is isomorphic to $\operatorname{Irr}(\tilde{B},[r])$ as $N_{F}(B)$-sets, where $\operatorname{Irr}(B,[r])=\operatorname{Irr}(B) \cap \operatorname{Irr}(G,[r])$ and $\operatorname{Irr}(\tilde{B},[r])=\operatorname{Irr}(\tilde{B}) \cap \operatorname{Irr}(\tilde{G},[r])$. Here $B \in \operatorname{Blk}(G)$ with cyclic defect groups, $\tilde{B}$ is the Brauer correspondent of $B$ in $\tilde{G}=N_{G}(\Omega(D))$, where $\Omega(D) \leqslant D$ is the unique subgroup of order $p$.

This follows by [6, Proposition 2.1].

Case (2) The corresponding result of Uno's refinement to the covering theorem, [10, Theorem 5.3], holds, that is, in the notation of [10, Theorem 5.3] $\operatorname{Irr}(B, \lambda,[r])$ is isomorphic to $\operatorname{Irr}(\tilde{B}, \lambda,[r])$ as $N_{F}(B)$-sets.

In the notation of Section 5 of [10], suppose $e(B)>1$. Then the degreepreserving bijections $\Psi: \operatorname{Irr}(\bar{B}) \rightarrow \operatorname{Irr}(B \mid \lambda)$ and $\tilde{\Psi}: \operatorname{Irr}(\tilde{B}) \rightarrow \operatorname{Irr}(\tilde{B} \mid \lambda)$ are uniquely determined, where $\Psi$ and $\tilde{\Psi}$ are given by [10, (5.8) and (5.9)].

If $\bar{\chi} \in \operatorname{Irr}(\bar{B})$ and $\chi=\Psi(\bar{\chi}) \in \operatorname{Irr}(B \mid \lambda)$, then $\chi(1)=\bar{\chi}(1)$ and

$$
r(\chi) \equiv \frac{|G|_{p^{\prime}}}{\chi(1)_{p^{\prime}}}=\frac{|\bar{G}|_{p^{\prime}}|Z|_{p^{\prime}}}{\bar{\chi}(1)_{p^{\prime}}} \equiv r(\bar{\chi})|Z|_{p^{\prime}} \quad(\bmod p) .
$$

Similarly, if $\overline{\tilde{\chi}} \in \operatorname{Irr}(\overline{\tilde{B}})$ and $\tilde{\chi}=\tilde{\Psi}(\overline{\tilde{\chi}}) \in \operatorname{Irr}(\tilde{B} \mid \lambda)$, then

$$
r(\tilde{\chi}) \equiv r(\overline{\tilde{\chi}})|Z|_{p^{\prime}} \quad(\bmod p) .
$$

Let $\Gamma$ be the $N_{F}(B)$-isomorphism of $\operatorname{Irr}(\bar{B})$ and $\operatorname{Irr}(\overline{\tilde{B}})$ such that $r(\bar{\chi}) \equiv \pm r(\overline{\tilde{\chi}})$ and $\Phi=\tilde{\Psi} \circ \Gamma \circ \Psi^{-1}$. As shown in the proof of [10, p. 956] $\Phi$ is an $N_{F}(B)$-isomorphism between $\operatorname{Irr}(B \mid \lambda)$ onto $\operatorname{Irr}(\tilde{B} \mid \lambda)$. But

$$
r(\chi) \equiv \pm r(\Phi(\chi)) \quad(\bmod p)
$$

so that $\Phi$ is an $N_{F}(B)$-isomorphism of $\operatorname{Irr}(B, \lambda,[r])$ onto $\operatorname{Irr}(\tilde{B}, \lambda,[r])$.

Suppose $e(B)=1$. Then $\chi_{i}(1)=\chi_{j}(1)$ and $\tilde{\chi}_{i}(1)=\tilde{\chi}_{j}(1)$ for any $\chi_{i}, \chi_{j} \in$ $\operatorname{Irr}(B \mid \lambda)$ and $\tilde{\chi}_{i}, \tilde{\chi}_{j} \in \operatorname{Irr}(\tilde{B} \mid \lambda)$ (cf. [10, p. 958]). It follows that $r\left(\chi_{i}\right) \equiv$ 
$r\left(\chi_{j}\right)(\bmod p)$ and $r\left(\tilde{\chi}_{i}\right) \equiv r\left(\tilde{\chi}_{j}\right)(\bmod p)$. In addition, there is an $N_{F}(B)$-isomorphism $\Gamma^{\prime}$ of $\operatorname{Irr}(B \mid \lambda)$ onto $\operatorname{Irr}(\tilde{B} \mid \lambda)$ (cf [10, p. 959]). Since $\Psi$ and $\tilde{\Psi}$ are degreepreserving and since there is a bijection $\Gamma$ of $\operatorname{Irr}(\bar{B})$ onto $\operatorname{Irr}(\overline{\tilde{B}})$, it follows that

$$
r(\chi) \equiv r\left(\Gamma^{\prime}(\chi)\right) \quad(\bmod p)
$$

so that $\Gamma^{\prime}$ is an $N_{F}(B)$-isomorphism of $\operatorname{Irr}(B, \lambda,[r])$ onto $\operatorname{Irr}(\tilde{B}, \lambda,[r])$.

Case (3) The corresponding result of Uno's refinement to the projective theorem, [10, Theorem 6.1], holds, that is, in the notation of [10, Theorem 6.1] $\operatorname{Irr}(B,[r])$ is isomorphic to $\operatorname{Irr}(\tilde{B},[r])$ as $N_{F}(B)$ sets.

In the notation of $[10,(6.15)]$, let $\Phi^{*}$ be the covering bijection of $\operatorname{Irr}(B)$ onto $\operatorname{Irr}\left(B^{*} \mid \zeta_{p}^{*}\right)$. Then by definition,

$$
r(\chi) \equiv r\left(\Phi^{*}(\chi)\right)\left|Z^{*}\right|_{p^{\prime}} \quad(\bmod p)
$$

for each $\chi \in \operatorname{Irr}(B)$.

Similarly, in the notation of $[10,(6.16)]$, let $\tilde{\Phi}^{*}$ be the covering bijection of $\operatorname{Irr}(\tilde{B})$ onto $\operatorname{Irr}\left(\tilde{B}^{*} \mid \zeta_{p}^{*}\right)$. Then

$$
r(\tilde{\chi}) \equiv r\left(\tilde{\Phi}^{*}(\tilde{\chi})\right)\left|Z^{*}\right|_{p^{\prime}} \quad(\bmod p)
$$

for each $\tilde{\chi} \in \operatorname{Irr}(\tilde{B})$.

It follows that the isomorphism $\Gamma^{*}$ of $\operatorname{Irr}\left(B^{*} \mid \zeta_{p}^{*}\right)$ onto $\operatorname{Irr}\left(\tilde{B}^{*} \mid \zeta_{p}^{*}\right)$ given by $[10$, (6.17)] is an $N_{F}(B)$ isomorphism of $\operatorname{Irr}\left(B^{*}, \zeta_{p}^{*},[r]\right)$ onto $\operatorname{Irr}\left(\tilde{B}^{*}, \zeta_{p}^{*},[r]\right)$. Thus there is an $N_{F}(B)$ isomorphism of $\operatorname{Irr}(B,[r])$ onto $\operatorname{Irr}(\tilde{B},[r])$.

Case (4) Now applying the proof given in [10, Section 7], we get the proof of Theorem 5.2.

\section{6. $p$-Central Extensions and $p$-Blocks}

We consider the relationship between $p$-blocks of central extension by a (cyclic) $p$ group and blocks of the original group. Given a central extension $\hat{X}$ of a finite group $X$ by a $p$-group $\hat{W}$ and corresponding blocks $A$ and $\hat{A}$ of $X$ and $\hat{X}$ respectively, we would like to have $k(X, A)=k(\hat{X}, \hat{A}, \lambda)$ for each $\lambda \in \operatorname{Irr}(\hat{W})$, and that similar equalities hold with respect to the more refined character counts considered in this article. This is studied in [10] when $A$ has cyclic defect groups, and in [25] when $\hat{A}$ has Abelian defect groups. Strong results concerning the role of fusion are obtained in [22], and it is these that we use, in particular to consider the case where $A$ has $p$ local rank one and generalised quaternion defect groups. We first need a definition from [22]. Note that although here we use $B$ and $G$ to represent blocks and groups, we are not constraining ourselves to Hypotheses 4.1.

Let $B$ be a block of a finite group $G$ with a Sylow $B$-subgroup $\left(D, b_{D}\right)$, and $Q$ a normal subgroup of $D$. Following [22], we say that the Brauer category of $B$ 
is $Q$-locally controlled by $\left(D, b_{D}\right)$ if, for any $B$-subgroup $\left(R, b_{R}\right) \leqslant\left(D, b_{D}\right)$ and any $g \in G$ such that $\left(R, b_{R}\right) \leqslant\left(D, b_{D}\right)^{g}:=g\left(D, b_{D}\right) g^{-1}$, there are elements $c \in C_{G}(R, Q)$ and $u \in D$ such that $g=c u$, where

$$
C_{G}(R, Q)=\{y \in G:[R, y] \subseteq Q\} .
$$

PROPOSITION 6.1. Let $G$ be a finite group and $B \in \operatorname{Blk}(G)$ a block of p-local rank one with a maximal Sylow B-subgroup $\left(D, b_{D}\right)$. Suppose that $L=O_{p}(G)$ is cyclic and central in $G$, and that we may write $D=L \times Q$ for some $Q$. Then:

(a) the Brauer category of $B$ is $Q$-locally controlled by $\left(D, b_{D}\right)$;

(b) if $\bar{B}$ be the image of the block $B$ in $G / O_{p}(Z(G))$ and $\lambda$ is a faithful linear character of $L$, then $\bar{B}$ is a block of $G / Z(G)$ with a defect group $D / L \simeq Q$, and there is a degree-preserving bijection between $\operatorname{Irr}(B, \lambda)$ and $\operatorname{Irr}(\bar{B})$.

Proof. (a) Note that since $\operatorname{prr}(B)=1$, it follows that $D / L \simeq Q$ is a TI subgroup of $G / Z(G)$. Since $L$ is cyclic, it possesses a faithful linear character $\lambda$. Let $\mu$ be the canonical extension of $\lambda$ to $D$ with $\operatorname{ker}(\mu)=Q$. If $\left(R, b_{R}\right)$ is a $B$-subgroup of $\left(D, b_{D}\right)$ with $\left(R, b_{R}\right) \leqslant\left(D, b_{D}\right)^{g}$ for some $g \in G$, then $C_{G}(L R)=C_{G}(R)$ and $C_{G}(L R, Q)=C_{G}(R, Q)$ and $\left(L R, b_{R}\right)$ is also a $B$-subgroup with $\left(L R, b_{R}\right) \leqslant$ $\left(D, b_{D}\right),\left(D, b_{D}\right)^{g}$. In particular we may assume that $L \leqslant R$. We may further assume that $L<R$, since otherwise $C_{G}(R, Q)=G$ and $g \in C_{G}(R, Q)$ trivially. Write $R=L \times R_{1}$ with $R_{1}=R \cap Q \neq 1$. Since $1 \neq R / L \leqslant D / L \cap(D / L)^{g L}$ and $D / L$ is TI, it follows that $g \in N_{G}(D)$. But then $\mu^{g}=\mu$, so $g$ normalizes $Q=\operatorname{ker}(\mu)$. Thus $R_{1}^{g} \leqslant Q^{g}=Q$ and $g \in C_{G}(R, Q)=C_{G}\left(R_{1}, Q\right)$. Hence by definition the Brauer category of $B$ is $Q$-locally controlled by $\left(D, b_{D}\right)$.

(b) $B$ can be viewed as a subalgebra of $\mathcal{O} G$, so $\bar{B}$ is a subalgebra of $\mathcal{O}(G / L)$. By part (a) and Theorem 7 of [22], $B_{\gamma} \cong \mathcal{O} L \otimes_{\mathcal{O}} \bar{B}_{\gamma}$, where $B_{\gamma}$ is the source algebra associated to a defect pointed group $D_{\gamma}$ corresponding to $\left(D, b_{D}\right)$, and $\bar{B}_{\gamma}$ is the image of $B_{\gamma}$ in $\mathcal{O}(G / L)$. Now $B_{\gamma}=i \mathcal{O} G i$ for $i \in \gamma$ and $\bar{B}_{\gamma}=\bar{i} \mathcal{O} \overline{G i}$, so $\bar{i}$ must necessarily be primitive and $\bar{B}_{\gamma}$ is a source algebra for $\bar{B}$. Hence $B$ is Morita equivalent to $B_{\gamma}$ and $\bar{B}$ to $\bar{B}_{\gamma}$.

As shown in the proof of [23, Theorem 5],

$$
B \simeq \mathcal{O} L \otimes_{\mathcal{O}} \bar{B}
$$

as algebras. (This can also be obtained by going through the results of [22] using $B$ instead of $B_{\gamma}$.) Proposition 6.1 follows.

Remark. The conditions of the above proposition are satisfied whenever a block $B \in \operatorname{Blk}(G)$ satisfies all of: (i) $\operatorname{plr}(B)=1$, (ii) $O_{p}(G)$ is cyclic and central in $G$, (iii) a defect group $D$ of $B$ is abelian or $D / O_{p}(G)$ has trivial Schur multiplier. Note that generalized quaternion and cyclic $p$-groups have trivial Schur multiplier. 
COROLLARY 6.2. Suppose that a block B satisfies the conditions of Proposition 6.1 and Conjecture 1.3 holds for the unique block $\bar{B}$ of $G / L$ corresponding to $B$. Then the conjecture holds for $B$.

Proof. Since $\operatorname{plr}(B)=1$ and $O_{p}(G) \leqslant Z(G)$, we must show that $k_{d}(G, B, \lambda$, $[r])=k_{d}\left(N_{G}(D), B, \lambda,[r]\right)$. But applying Proposition 6.1 to $B$ and its Brauer correspondent in $N_{G}(D)$ gives

$$
\begin{aligned}
k_{d}(G, B, \lambda,[r]) & =k_{d^{\prime}}(G / L, \bar{B},[r])=k_{d^{\prime}}\left(N_{G}(D) / L, \bar{B},[r]\right) \\
& =k_{d}\left(N_{G}(D), B, \lambda,[r]\right),
\end{aligned}
$$

since the conjecture holds for $\bar{B}$ (here $d^{\prime}=d-\log _{p}(|L|)$ ).

\section{Checking the Conjecture}

We have shown in Section 4.7 that in proving Theorem 1.4 it suffices to check Conjecture 1.3 for TI defect blocks of groups $G$ with $F(G) \leqslant Z(G)$ and $F^{*}(G) / Z(G)$ non-abelian simple (and $Z(G) \leqslant G^{\prime}$ with $Z(G)$ cyclic). We still keep the notation of Hypotheses 4.1. A complete list of such blocks is obtained in [4], which we summarise here. Since there is a one-to-one correspondence between blocks of $G$ and blocks of $G / O_{p}(Z(G))$ which preserves the $p$-local rank and takes defect groups to their quotient modulo $O_{p}(Z(G))$, it suffices to classify those blocks of groups with $O_{p}(Z(G))=1$.

THEOREM 7.1. Let $B$ be a block of p-local rank one of a finite group $G$ for which $F(G) \leqslant Z(G)$ and $F^{*}(G) / Z(G)$ is non-Abelian simple. Let $\bar{B}$ be the unique p-block of $G / O_{p}(Z(G))$ corresponding to $B$. Then $\bar{B}$ is one of the following:

(a) a block with cyclic or generalised quaternion or Klein-four defect groups;

(b) a 2- or 3-block with maximal defect groups;

(c) the unique block of $O^{\prime} N$, mathrm Aut $\left(O^{\prime} N\right), 2 . S u z$, mathrmAut (Suz) with defect groups of the form $C_{3} \times C_{3}$;

(d) a 5-block of maximal defect of 3.McL or mathrmAut $(M c L)$;

(e) the principal 11-block of $J_{4}$;

(f) a p-block of maximal defect of a $p^{\prime}$-central extension of a group $X$ with $Y \leqslant$ $X \leqslant \operatorname{Aut}(Y)$, where $(p,[X: Y])=1$ and $Y \cong \operatorname{PSL}_{2}\left(p^{m}\right)$ or $\operatorname{PSU}_{3}\left(p^{m}\right)$ for some $m>1$;

(g) the principal 5-block of ${ }^{2} F_{4}(2)^{\prime},{ }^{2} F_{4}(2)$ or mathrmAut $\left({ }^{2} B_{2}(25)\right)$.

There is a subtlety which we address here. Note that most of the above cases occur as central extensions of some group of automorphisms of a non-Abelian simple group. It may so happen that the Schur multiplier of the automorphism 
group of a simple group is different from that of the simple group itself. We deal with this in the same way as in [11]. First, observe that if the outer automorphism group is cyclic, then there is no problem.

LEMMA 7.2. Let $Y \unlhd X$, where $Y$ is perfect and $X / Y$ is cyclic. Then the Schur multiplier of $X$ is a quotient of that of $Y$.

Proof. This follows directly from [11, 3.4].

This applies to cases (c), (d), (e), (g). We examine case (a) by general methods in which Schur multipliers do not play a role, and (b) will follow from [11]. This leaves case (f), which we address as follows.

Suppose that $X$ has TI Sylow $p$-subgroups, $O_{p}(X)=1, Z(X)$ is cyclic and contained in $X^{\prime}$, and that there is $Y \unlhd X$ such that $Y$ is a central extension of a non-Abelian simple group by $Z(X)$. Suppose also that $X / Y$ is a direct product of two cyclic $p^{\prime}$-groups, and let $Y \unlhd T \unlhd X$, where $T / Y$ and $X / T$ are cyclic. Write $W$ for the central extension of $T / Z(G)$ by $T^{\prime} \cap Z(G)$ such that $W \leqslant T$. We have $Z\left(Y^{\prime}\right) \leqslant Z(W) \leqslant Z(G)$. The proof of the following is based on $[7,3.6]$.

LEMMA 7.3. Let $X$ be as above. Then there is a finite group $H$ satisfying

(i) $Z(H) \leqslant Y^{\prime} \unlhd H$,

(ii) $O_{p^{\prime}}(H)=Z(H) \leqslant H^{\prime}, Z(H)$ is cyclic and $O_{p}(H)=1$,

(iii) $C_{H}\left(Y^{\prime}\right)=Z(H)=Z\left(Y^{\prime}\right)$,

(iv) $H / Y^{\prime}$ is a $p^{\prime}$-group,

(v) Conjecture 1.3 holds for $X$ if and only if it holds for $H$.

Proof. This is almost identical to the proof of [11, 3.6, 3.7]. The difference is that our character count is more refined than that for DPC, however, we have already seen that the Clifford-theoretic techniques used in the proof of $[11,3.6]$ are compatible with the residues used in the statement of Conjecture 1.3.

PROPOSITION 7.4. Let $G$ be a finite group and $B \in \mathrm{Blk}(G)$ with a defect group $D$. Suppose $G, D$ are given as follows.

(i) $G=3 . M c L$ or $\operatorname{Aut}(M c L)=M c L .2$ and $D=5_{+}^{1+2} \in \operatorname{Syl}_{5}(G)$.

(ii) $G={ }^{2} F_{4}(2)^{\prime}$ or ${ }^{2} F_{4}(2)$ and $D=C_{5} \times C_{5} \in \operatorname{Syl}_{5}(G)$.

Table I. The normalizers $N=N_{G}(D)$

\begin{tabular}{llll}
\hline$G$ & $N$ & $G$ & $N$ \\
\hline $3 . M c L$ & $3 \times 5_{+}^{1+2}: 3: 8$ & $M c L .2$ & $5_{+}^{1+2}: 3: 8.2$ \\
${ }^{2} F_{4}(2)^{\prime}$ & $5^{2}: 4 . A_{4}$ & ${ }^{2} F_{4}(2)$ & $5^{2}: 4 . S_{4}$ \\
${ }^{2} B_{2}(25) .5$ & $25: 20$ & $J_{4}$ & $11_{+}^{1+2}:\left(5 \times 2 . S_{4}\right)$ \\
\hline
\end{tabular}


Table II. The characters of $\operatorname{Irr}(N, B)$

\begin{tabular}{ll}
\hline$N$ & $\operatorname{Irr}(N, B)$ \\
\hline $3 \times 5_{+}^{1+2}: 3: 8$ & $\{8 \times 1,4 \times 2,6 \times 20,1 \times 24\}$ \\
$5_{+}^{1+2}: 3: 8.2$ & $\{8 \times 1,10 \times 2,4 \times 20,2 \times 24,2 \times 40\}$ \\
$5^{2}: 4 . A_{4}$ & $\{6 \times 1,6 \times 2,2 \times 3,2 \times 24\}$ \\
$5^{2}: 4 . S_{4}$ & $\{4 \times 1,6 \times 2,4 \times 3,2 \times 4,4 \times 24\}$ \\
$25: 20$ & $\{20 \times 1,5 \times 4,1 \times 20\}$ \\
$11_{+}^{1+2}:\left(5 \times 2 . S_{4}\right)$ & $\{10 \times 1,15 \times 2,10 \times 3,5 \times 4,3 \times 110,2 \times 120,3 \times 220,1 \times 330\}$ \\
\hline
\end{tabular}

Table III. The values $k_{d}(G, B,[r])$

\begin{tabular}{lrrrrl}
\hline Defect $d$ & 3 & 3 & 2 & 2 & otherwise \\
\hline value $r$ & 2 & 1 & 2 & 1 & otherwise \\
\hline$k_{d}(3 . M c L, B,[r])$ & 9 & 4 & 6 & 0 & 0 \\
$k_{d}(M c L .2, B,[r])$ & 10 & 10 & 4 & 2 & 0 \\
$k_{d}\left({ }^{2} F_{4}(2)^{\prime}, B,[r]\right)$ & 0 & 0 & 8 & 8 & 0 \\
$k_{d}\left({ }^{2} F_{4}(2), B,[r]\right)$ & 0 & 0 & 10 & 10 & 0 \\
$k_{d}\left({ }^{2} B_{2}(25) .5, B,[r]\right)$ & 0 & 25 & 0 & 1 & 0 \\
\hline
\end{tabular}

Table IV. The values $k_{d}\left(J_{4}, B_{0},[r]\right)$

\begin{tabular}{lrrrrrrl}
\hline Defect $d$ & 3 & 3 & 3 & 2 & 2 & 2 & otherwise \\
\hline value $r$ & 2 & 3 & 5 & 1 & 2 & 3 & otherwise \\
\hline$k_{d}\left(J_{4}, B_{0},[r]\right)$ & 12 & 10 & 5 & 3 & 3 & 1 & 0 \\
\hline
\end{tabular}

(iii) $G=\operatorname{Aut}\left({ }^{2} B_{2}(25)\right)={ }^{2} B_{2}(25) .5$ and $D=5_{+}^{1+2} \in \operatorname{Syl}_{5}(G)$.

(iv) $G=J_{4}$ and $D=11_{+}^{1+2} \in \operatorname{Syl}_{11}(G)$.

Thus $B$ is the principal block $B_{0}=B_{0}(G)$ of $G$ except when $G=3 . M c L$, in which case $B$ is one of the three blocks $B_{0}, B_{1}, B_{2}$ with a defect group $D$. Then Conjecture 1.3 holds for $B$.

Proof. If $N=N_{G}(D)$, then by [8], the structure of $N$ is given in Table I.

Note that $N$ is a maximal subgroup of $G$ and that its character table can be found in the library of character tables distributed with GAP, except when $N=$ $N_{F_{F_{4}(2)}}(D)=5^{2}: 4 . S_{4}$ or $N=N_{B_{2}(25) .5}(D)=25: 20$. In the latter case, $\operatorname{Irr}(N)$ is given by [11, p.635], and in the former case, it can be calculated easily using the table of $N_{2} F_{4}(2)^{\prime}(D)=5^{2}: 4$. $A_{4}$. If $G=3 . M c L$ and $B=B_{i}$ for $0 \leqslant i \leqslant 2$, 
then there is a degree-preserving bijection of $\operatorname{Irr}\left(N, B_{i}\right)$ onto $\operatorname{Irr}\left(N, B_{0}\right)$, since $N=$ $3 \times N_{M c L}(D)$. The characters of $\operatorname{Irr}(N, B)$ are given in Table II.

The character table of $G$ is given by [8]. Suppose $p=5$. Then the values $k_{d}(G, B,[r])$ are given in Table III.

If $p=11$, then the values $k_{d}\left(J_{4}, B_{0},[r]\right)$ are given in Table IV.

Now Proposition 7.4 follows by Tables II, III and IV.

In order to verify Conjecture 1.3 for case (f) of Theorem 7.1 we make use of the computations of numbers of fixed points under the action of automorphisms on the irreducible characters of these groups. As with [11], in order to do so we must use a strengthened version of [7, 2.3]. Since the proof is essentially the same, with the observation that all the methods involved respect the residues used in Conjecture 1.3, we omit it.

Let $O$ be a finite group acting on a finite set $\Omega$ and $f(\Omega, t)$ the number of elements of $\Omega$ fixed by $t$. Note that if $O$ acts on another set $\Omega^{\prime}$, then there is a bijection $\Psi$ from $\Omega$ to $\Omega^{\prime}$ such that $\Psi(x)^{\tau}=\Psi\left(x^{\tau}\right)$ if and only if $f(\Omega, w)=$ $f\left(\Omega^{\prime}, w\right)$ for each $w \in\langle\tau\rangle$, where $\tau$ is a fixed element of $O$.

LEMMA 7.5. Suppose $X$ is a finite group, $H \leqslant G \unlhd X$ with $X / G$ a cyclic $p^{\prime}$-group. Suppose $y \in X$ generates $X / G$ and normalizes $H$ and that $|X: G|=$ $|H\langle y\rangle: H|$. Let $B$ be a union of p-blocks of $G$ and $b$ a union of p-blocks of $H$ such that $y$ stabilizes $B$ and $b$. Suppose for each subgroup $C$ of $\langle y\rangle$, $f\left(\operatorname{Irr}_{d}(G, B,[r]), C\right)=f\left(\operatorname{Irr}_{d}(H, b,[r]), C\right)$. Let $B_{1}$ be the union of all p-blocks of $X$ covering $B$ and $b_{1}$ the union of all p-blocks of $H\langle y\rangle$ covering $b$. Then

$$
\left|\operatorname{Irr}_{d}\left(X, B_{1},[r]\right)\right|=\left|\operatorname{Irr}_{d}\left(H\langle y\rangle, b_{1},[r]\right)\right| .
$$

PROPOSITION 7.6. Let $B$ be a p-block of maximal defect of a $p^{\prime}$-central extension $G$ of a group $X$ with $Y \leqslant X \leqslant \operatorname{Aut}(Y)$, where $(p,[X: Y])=1$ and $Y \cong \operatorname{PSL}_{2}\left(p^{m}\right)$ or $\mathrm{PSU}_{3}\left(p^{m}\right)$ for some $m>1$. Then Conjecture 1.3 holds for $B$.

Proof. If $p=2$ or 3 , then Conjecture 1.3 is equivalent to Conjecture 1.1, so that Proposition 7.6 follows by [11] as $G$ has $p$-local rank one.

Suppose $p \geqslant 5$ and $q=p^{m}$ (so $O_{p}(G)=1$ ). By Lemma 7.3 it suffices to assume that the preimage $K$ of $Y$ in $G$ is perfect, i.e., $K=\mathrm{SL}_{2}(q)$ or $\mathrm{SU}_{3}(q)$ (since by considering the principal block we obtain the conjecture for the case where the centre is trivial).

Let $U$ be the Sylow $p$-subgroup of $K$ consisting of unit lower triangular matrices of $K, W=N_{K}(U)$ a Borel subgroup of $K$, so that $W=U \rtimes C_{q^{n-1}-1}$, where $n=2$ or 3 according as $K=\mathrm{SL}_{2}(q)$ or $\mathrm{SU}_{3}(q)$. In addition, let $\delta=\operatorname{gcd}(n, q-\epsilon)$ and $Z=Z(K)$, so that $|Z|=\delta$, where $\epsilon=1$ or -1 according as $K=\mathrm{SL}_{2}(q)$ or $\mathrm{SU}_{3}(q)$.

Case (1) If $K=\mathrm{SU}_{3}(q)$, then the character tables of both $W$ and $K$ are given by [16, Tables 2.1 and 3.1]. In particular, $|\operatorname{Irr}(W)|=q^{2}+q+\delta^{2}$ and $|\operatorname{Irr}(K)|=$ $q^{2}+q+1+\delta^{2}$. If $\delta=1$, then the principal blocks $B_{0}=B_{0}(K)$ and $b_{0}=B_{0}(W)$ 
are the only blocks of $K$ and $W$ with maximal defect, respectively. If $\delta=3$, then $K$ and $W$ both have three blocks $B_{i}$ and $b_{i}$, respectively with maximal defect, where $\mu_{i} \in \operatorname{Irr}(Z)$ with $\mu_{0}=1$, and $B_{i}$ and $b_{i}$ cover the block $B\left(\mu_{i}\right)$ of $Z$ containing $\mu_{i}$. Thus $B_{0}=B_{0}(K), B_{1}$ and $B_{2}$ are conjugate under the complex conjugation.

Suppose $\delta=3$. In the notation of [16, Tables 2.1 and 3.1], let

$$
X_{K}=\left\{\chi_{(q-1)\left(q^{2}-q+1\right) / 3}^{(u)}, \chi_{(q-1)(q+1)^{2} / 3}^{(u, v)}: 0 \leqslant u \leqslant 2, v=1,2\right\} \subseteq \operatorname{Irr}(K)
$$

and $X_{W}=\left\{\vartheta_{\left(q^{2}-1\right) / 3}^{(u, v)}: 0 \leqslant u, v \leqslant 2\right\} \subseteq \operatorname{Irr}(W)$. Then $\left|X_{K}\right|=\left|X_{W}\right|=9$ and $\left|\operatorname{Irr}\left(B_{i}\right) \cap X_{K}\right|=\left|\operatorname{Irr}\left(b_{i}\right) \cap X_{W}\right|=3$ for each $i$.

For $\chi \in(\operatorname{Irr}(K) \backslash\{\mathrm{St}\}) \cup \operatorname{Irr}(W)$,

$$
r(\chi) \equiv \begin{cases} \pm 1(\bmod p) & \text { if } \delta=1 \text { or } \delta=3 \text { and } \chi \notin X_{K} \cup X_{W} \\ \pm 3^{-1}(\bmod p) & \text { if } \chi \in X_{K} \cup X_{W}\end{cases}
$$

where St is the Steinberg character of $K$.

Suppose $L=K$ or $W$. Let $b_{i}(L)=B_{i}$ or $b_{i}(L)=b_{i}$ according as $L=K$ or $W$, and when $\delta=3$, let

$$
\varphi_{L}^{(u, v)}= \begin{cases}\vartheta_{\left(q^{2}-1\right) / 3}^{(v, u)} & \text { if } L=W, \\ \chi_{(q-1)\left(q^{2}-q+1\right) / 3}^{(u)} & \text { if } L=K \text { and } v=0, \\ \chi_{\left.(q-1)(q+1)^{2}\right) / 3}^{(u, v)} & \text { if } L=K \text { and } v=1,2 .\end{cases}
$$

If $\delta=1$, then $r(\chi) \equiv \pm 1(\bmod p)$ and $d(\chi)=2 m$ or $3 m$ for each $\chi \in$ $\operatorname{Irr}\left(b_{0}(L)\right)$, so that $\operatorname{Irr}_{d}\left(b_{0}(L), \lambda,[1]\right)=\operatorname{Irr}_{d}\left(b_{0}(L), \lambda\right)$.

Suppose $\delta=3$. Then $\operatorname{Irr}_{d}\left(L, B_{i}, \lambda,[1]\right)=\operatorname{Irr}_{d}\left(L, B_{i}, \lambda\right)$ except when $d=3 m$, in which case,

$$
\operatorname{Irr}_{3 m}\left(b_{i}(L),\left[3^{-1}\right]\right)=X_{L} \cap b_{i}(L)=\left\{\varphi_{L}^{(u, i)}\right\},
$$

$\operatorname{Irr}_{3 m}\left(b_{i}(L),[1]\right)=\operatorname{Irr}_{3 m}\left(b_{i}(L)\right) \backslash \operatorname{Irr}_{3 m}\left(b_{i}(L),\left[3^{-1}\right]\right)$. Note that $b_{0}(L)$ is the unique block of $L$ with maximal defect covering the trivial character of $Z$. Moreover, if $\bar{\chi}$ is the complex dual character of $\chi$, then $\chi \mapsto \bar{\chi}$ is a bijection from $\operatorname{Irr}\left(b_{1}(L)\right)$ onto $\operatorname{Irr}\left(b_{2}(L)\right)$.

Note a semisimilarity $t \in \Gamma U_{3}(q)$ stabilizing $b_{i}(L)$ always stabilizes $\operatorname{Irr}_{d}\left(b_{i}(L)\right.$, $\lambda$, $[r])$, since $t$ normalizes $Z$ and preserves degree.

Let $x$ be the diagonal element of $U_{3}(q)$ given by [7, p. 436], so that $x$ induces a diagonal automorphism on $K$ and $x^{3} \in K$. In the notation of [16] (cf. [7, Table 2]), $x$ normalizes $L$ and fixes each conjugacy class $C$ of $L$ except when $C=C_{3}^{(k, l)}$ or $B_{3}^{(k, l)}$ according as $L=K$ or $W$, in which case $\delta=3$ and $x$ fuses $\left\{C_{3}^{(k, l)}: 0 \leqslant\right.$ $\ell \leqslant \delta\}$ or $\left\{B_{3}^{(k, l)}: 0 \leqslant \ell \leqslant \delta\right\}$ (see the remark of [16, p. 566]). It follows by the character tables of $K$ and $W$ that $x$ fixes each character of $\operatorname{Irr}_{d}\left(b_{i}(L),[r]\right)$ except when $d=3 m$ and $r \equiv \pm 3^{-1}(\bmod p)$, in which case $\delta=3$ and $x$ fuses characters in $\operatorname{Irr}_{3 m}\left(b_{i}(L),\left[3^{-1}\right]\right)$ (cf. Remarks on [16, p. 571]). In particular, 


$$
(\bar{\chi})^{w}=\overline{\left(\chi^{w}\right)}
$$

for each $\chi \in \operatorname{Irr}\left(b_{1}(L)\right)$ and $w=x$.

Suppose $y$ is a semisimilarity inducing the field automorphism $\tau^{e}$ on $K$ and stabilizing $b_{i}(L)$, where $\tau$ is the Frobenius automorphism of $K$. Thus $\left(C_{3}^{(0, l)}\right)^{y}=$ $C_{3}^{\left(0, p^{e} l\right)}$ and $\left(B_{3}^{(0, l)}\right)^{y}=B_{3}^{\left(0, p^{e} l\right)}$, so that $y$ fixes $\varphi_{L}^{(0, i)}$, and $y$ fuses $\varphi_{L}^{(1, i)}$ and $\varphi_{L}^{(2, i)}$ if and only if $y$ fuses $C_{3}^{(0,1)}$ and $C_{3}^{(0,2)}$, or $B_{3}^{(0,1)}$ and $B_{3}^{(0,2)}$. Since $B_{3}^{(0, l)} \subseteq C_{3}^{(0, l)}$ for each $l$, it follows that $y$ fuses $\varphi_{K}^{(1, i)}$ and $\varphi_{K}^{(2, i)}$ if and only if $y$ fuses $\varphi_{W}^{(1, i)}$ and $\varphi_{W}^{(2, i)}$. Moreover, by the character tables of $K$ and $W$, (7.1) holds for $w=y$, so that it holds for any $w \in \Gamma U_{3}(q)$ centralizing $Z$.

If follows that for any $t \in \Gamma U_{3}(q)$ centralizing $Z$, any integers $d, r$ and any $\lambda \in \operatorname{Irr}(Z)$,

$$
f\left(\operatorname{Irr}_{d}\left(b_{1}(L), \lambda,[r]\right), t\right)=f\left(\operatorname{Irr}_{d}\left(b_{2}(L), \lambda,[r]\right), t\right) .
$$

As shown in [7, p. 455], $f\left(\operatorname{Irr}_{3 m}\left(B_{0}\right), t\right)=f\left(\operatorname{Irr}_{3 m}\left(b_{0}\right), t\right)$ and in addition, if $\delta=3$, then

$$
f\left(\operatorname{Irr}_{3 m}\left(B_{1}\right), t\right)+f\left(\operatorname{Irr}_{3 m}\left(B_{2}\right), t\right)=f\left(\operatorname{Irr}_{3 m}\left(b_{1}\right), t\right)+f\left(\operatorname{Irr}_{3 m}\left(b_{2}\right), t\right) .
$$

By (7.2),

$$
f\left(\operatorname{Irr}_{d}\left(B_{i}\right), t\right)=f\left(\operatorname{Irr}_{d}\left(b_{i}\right), t\right)
$$

for any $i$ when $d=3 m$.

By [7, Table 1] and the Brauer permutation lemma, $f\left(\operatorname{Irr}\left(B_{0}\right), t\right)=f\left(\operatorname{Irr}\left(b_{0}\right), t\right)$ and if $\delta=3$, then

$$
f\left(\operatorname{Irr}\left(B_{1}\right), t\right)+f\left(\operatorname{Irr}\left(B_{2}\right), t\right)=f\left(\operatorname{Irr}\left(b_{1}\right), t\right)+f\left(\operatorname{Irr}\left(b_{2}\right), t\right) .
$$

It follows by this and (7.2) that

$$
f\left(\operatorname{Irr}\left(B_{i}\right), t\right)=f\left(\operatorname{Irr}\left(b_{i}\right), t\right)
$$

for each $i$. But each character of $\operatorname{Irr}\left(b_{i}(L)\right)$ either has defect $3 m$ or $2 m$ and (7.3) holds when $d=3 m$, so (7.3) holds for any $d$ and this implies that

$$
f\left(\operatorname{Irr}_{d}\left(B_{i}, \lambda,[r]\right), t\right)=f\left(\operatorname{Irr}_{d}\left(b_{i}, \lambda,[r]\right), t\right)
$$

when $d \neq 3 m$, since $r(\chi) \equiv \pm 1(\bmod p)$ for each $\chi \in \operatorname{Irr}_{2 m}\left(B_{i}\right) \cup \operatorname{Irr}_{2 m}\left(b_{i}\right)$.

Suppose $d=3 m$. By reordering, we may suppose the action of $x$ on $\operatorname{Irr}_{3 m}\left(b_{i}(L)\right.$, $\left.\left[3^{-1}\right]\right)$ is given by the permutation $\left(\varphi_{L}^{(0, i)}, \varphi_{L}^{(1, i)}, \varphi_{L}^{(2, i)}\right)$, with $\varphi_{L}^{(0, i)}$ fixed by $y$, where $x$ and $y$ are given as before. Given $i$, define $\phi: \operatorname{Irr}_{3 m}\left(B_{i},\left[3^{-1}\right]\right) \rightarrow \operatorname{Irr}_{3 m}\left(b_{i},\left[3^{-1}\right]\right)$ by $\phi\left(\varphi_{K}^{(u, i)}\right)=\varphi_{W}^{(u, i)}$. Then

$$
\phi\left(\chi^{t}\right)=\phi(\chi)^{t}
$$


for $t=x$ and $y$, so that it holds for any $t \in \Gamma U_{3}(q)$ stabilizing $b_{i}$. In particular, (7.5) holds when $d=3 m$ and $r=3^{-1}$. It follows by (7.3) that (7.5) holds for $d=3 m$ and $r=1$, so that it holds for any $d, \lambda$ and $r$.

If $G / K$ is cyclic, then Proposition 7.6 follows by (7.5) and Lemma 7.5.

Suppose $G / K$ is non-cyclic. We may suppose $x, y \in G$. Applying the remark given before Lemma 7.5 with $\tau=y$, we have a bijection $\Phi: \operatorname{Irr}_{d}\left(B_{i}, \lambda,[r]\right) \rightarrow$ $\operatorname{Irr}_{d}\left(b_{i}, \lambda,[r]\right)$ such that

$$
\Phi\left(\chi^{t}\right)=\Phi(\chi)^{t}
$$

for $t=y$ and $\chi \in \operatorname{Irr}_{d}\left(B_{i}, \lambda,[r]\right)$. Since $x$ fixes each character in the complement of $\operatorname{Irr}_{3 m}\left(b_{i}(L),\left[3^{-1}\right]\right)$ in $\operatorname{Irr}(L)$, it follows that (7.6) holds when $t=x$ if $d \neq 3 m$ or $r \not \equiv \pm 3^{-1}(\bmod p)$. If $d=3 m$ and $r \equiv \pm 3^{-1}(\bmod p)$, then we may choose $\Phi=\phi$ given above, so that (7.6) still holds when $t=y$. Thus (7.6) holds for any $t \in \Gamma U_{3}(q)$ stabilizing $b_{i}$.

Let $b_{i}^{\prime}(L)$ be the block of $L\langle x\rangle$ covering $b_{i}(L)$. For any $\chi^{\prime} \in b_{i}^{\prime}(L), d\left(\chi^{\prime}\right)=2 m$ or $3 m$ and $r\left(\chi^{\prime}\right) \equiv \pm 3(\bmod p)$.

Let $\theta$ be the inflation to $L\langle x\rangle$ of a fixed non-trivial character of $\operatorname{Irr}(L\langle x\rangle / L)$. Suppose $\chi_{L} \in \operatorname{Irr}\left(b_{i}(L)\right)$. If $\chi_{L} \notin \operatorname{Irr}_{3 m}\left(b_{i}(L),\left[3^{-1}\right]\right)$, then $\chi_{L}$ has three extensions $\chi_{L} \otimes \theta^{l}, l=0,1,2$ in $\operatorname{Irr}\left(b_{i}^{\prime}(L)\right)$. Define $\Phi^{\prime}\left(\chi_{K} \otimes \theta^{l}\right)=\Phi\left(\chi_{W}\right) \otimes \theta^{l}$. Then

$$
\Phi^{\prime}\left(\chi_{K} \otimes \theta^{l}\right)^{y}=\Phi\left(\chi_{W}^{y}\right) \otimes\left(\theta^{p^{e} l}\right)=\Phi^{\prime}\left(\left(\chi_{K} \otimes \theta^{l}\right)^{y}\right) .
$$

If $\chi_{L} \in \operatorname{Irr}_{3 m}\left(b_{i}(L),\left[3^{-1}\right]\right)$, then $\chi_{L}$ has a unique extension $\chi_{L}^{\prime}$ in $\operatorname{Irr}\left(b_{i}^{\prime}(L)\right)$ and $\chi_{L}^{\prime}$ is the unique character in $\operatorname{Irr}\left(b_{i}^{\prime}(L)\right)$ covering all characters of $\operatorname{Irr}_{3 m}\left(b_{i}(L),\left[3^{-1}\right]\right)$. In particular, $y$ fixes $\chi_{L}^{\prime}$. Set $\Phi^{\prime}\left(\chi_{K}^{\prime}\right)=\chi_{W}^{\prime}$. Then $\Phi^{\prime}$ is an isomorphism from $\operatorname{Irr}_{d}\left(b_{i}^{\prime}(K), \lambda,[r]\right)$ onto $\operatorname{Irr}_{d}\left(b_{i}^{\prime}(W), \lambda,[r]\right)$ as $\langle y\rangle$-sets, since $r\left(\Phi^{\prime}\left(\chi^{\prime}\right)\right) \equiv r\left(\chi^{\prime}\right)$ $(\bmod p)$. In particular,

$$
f\left(\operatorname{Irr}_{d}\left(b_{i}^{\prime}(K), \lambda,[r]\right), t\right)=f\left(\operatorname{Irr}_{d}\left(b_{i}^{\prime}(W), \lambda,[r]\right), t\right)
$$

for any $t \in\langle y\rangle$. Now Proposition 7.6 follows by Lemma 7.5.

Case (2) Let $K=\mathrm{SL}_{2}(q)$. Then

$$
\begin{aligned}
\operatorname{Irr}(K)= & \left\{\chi_{1}, \chi_{q}, \chi_{q+1}^{(u)}, \chi_{(q-1)}^{(v)}, \chi_{(q+1) / 2}^{(a)}, \chi_{(q-1) / 2}^{(a)}:\right. \\
& \left.1 \leqslant u \leqslant \frac{q-3}{2}, 1 \leqslant v \leqslant \frac{q-1}{2}, a=1,2\right\}
\end{aligned}
$$

and $|\operatorname{Irr}(K)|=q+4$, where $\chi_{1}(1)=1, \chi_{q}(1)=q$ and $\chi_{i}^{(l)}(1)=i$ for all $i, l$. In addition, $Z \leqslant \operatorname{ker}\left(\chi_{(q-v) / 2}^{(a)}\right)$ if and only if $4 \mid(q-v)$, where $v=-1$ or 1 .

Let $\operatorname{Irr}(U)=\left\{\eta_{1}^{(j)}: 0 \leqslant j \leqslant q\right\}$, where $\eta_{1}^{(0)}$ is the trivial character of $U$. Thus $I_{W}\left(\eta_{1}^{(j)}\right)=W$ or $U Z$ according as $j=0$ or $j \neq 0$. It follows by Clifford theory that

$$
\operatorname{Irr}(W)=\left\{\psi_{1}^{(j)}, \psi_{(q-1) / 2}^{(a)}: 1 \leqslant j \leqslant q-1,1 \leqslant a \leqslant 4\right\},
$$


so that $|\operatorname{Irr}(W)|=q+3$, where $\psi_{i}^{(l)}(1)=i$. Moreover, there are exactly two characters $\psi=\psi_{(q-1) / 2}^{(a)}$ with $Z \leqslant \operatorname{ker}(\psi)$. Suppose $\psi_{(q-1) / 2}^{(1)}$ and $\psi_{(q-1) / 2}^{(2)}$ cover $\eta \otimes 1 \in \operatorname{Irr}(U \times Z)$, where $\eta$ is a fixed generator of $\operatorname{Irr}(U)$.

Let $X_{W}=\left\{\psi_{(q-1) / 2}^{(a)}: 1 \leqslant a \leqslant 4\right\}$ and $X_{K}=\left\{\chi_{(q+1) / 2}^{(a)}, \chi_{(q-1) / 2}^{(a)}: a=1,2\right\}$. Then $\left|X_{K}\right|=\left|X_{W}\right|=4$ and for $\chi \in\left(\operatorname{Irr}\left(\mathrm{SL}_{2}(q)\right) \backslash\{\mathrm{St}\}\right) \cup \operatorname{Irr}(W)$,

$$
r(\chi) \equiv \begin{cases} \pm 1(\bmod p) & \text { if } \chi \notin X_{K} \cup X_{W} \\ \pm 2^{-1}(\bmod p) & \text { if } \chi \in X_{K} \cup X_{W}\end{cases}
$$

For each $\mu_{i} \in \operatorname{Irr}(Z)$ with $\mu_{0}=1, \mathrm{SL}_{2}(q)$ and $W$ both have exactly one block $B_{i}$ and $b_{i}$ with maximal defect and covering the block $B\left(\mu_{i}\right)$ of $Z$ containing $\mu_{i}$, and moreover, $\left|X_{K} \cap B_{i}\right|=\left|X_{W} \cap b_{i}\right|=2$.

Suppose $L=K$ or $W$. Let $b_{i}(L)=B_{i}$ or $b_{i}$ according as $L=K$ or $W$. Each $\chi \in \operatorname{Irr}\left(b_{i}(L)\right)$ has defect $m$, so $\operatorname{Irr}_{m}\left(b_{i}(L),\left[2^{-1}\right]\right)=X_{L} \cap b_{i}(L)$ and

$$
\operatorname{Irr}_{m}\left(b_{i}(L),[1]\right)=\operatorname{Irr}\left(b_{i}(L)\right) \backslash \operatorname{Irr}_{m}\left(b_{i}(L),\left[2^{-1}\right]\right) .
$$

Suppose $x=\operatorname{diag}\left\{\alpha,-\alpha^{-1}\right\}$ with $\alpha$ a generator of $G F(q)^{\times}$. Then $x^{2} \in K, x$ induces the diagonal automorphism of $K$ and stabilizes $W$. Since the irreducible characters of $\mathrm{GL}_{2}(q)$ have degrees $1, q, q \pm 1$, it follows that $x$ fuses the characters in $\left\{\chi_{(q \pm 1) / 2}^{(1)}, \chi_{(q \pm 1) / 2}^{(2)}\right\}$ and stabilizes other characters. Similarly, it follows from the degrees of irreducible characters of a Borel subgroup of $\mathrm{GL}_{2}(q)$ that $x$ fuses the characters in each set $\left\{\psi_{(q-1) / 2}^{(1)}, \psi_{(q-1) / 2}^{(2)}\right\}$ and $\left\{\psi_{(q-1) / 2}^{(3)}, \psi_{(q-1) / 2}^{(4)}\right\}$, and fixes the other characters in $\operatorname{Irr}(W)$. Thus (7.5) holds when $t=x$.

Suppose $y \in \Gamma L_{2}(q)$ induces the standard Frobenius automorphism of $K$. Let $C^{(u)}$ for $1 \leqslant u \leqslant 4$ be the 4 nonsemisimple classes of $L$ with representatives,

$$
\left(\begin{array}{ll}
1 & 0 \\
1 & 1
\end{array}\right),\left(\begin{array}{cc}
1 & 0 \\
\alpha & 1
\end{array}\right),\left(\begin{array}{cc}
-1 & 0 \\
1 & -1
\end{array}\right),\left(\begin{array}{cc}
-1 & 0 \\
\alpha & -1
\end{array}\right)
$$

Thus $y$ fixes each $C^{(u)}$.

If $e \mid m$, then $C_{K}\left(y^{e}\right)=\mathrm{SL}_{2}\left(p^{e}\right)$ and $C_{W}\left(y^{e}\right)$ is a Borel subgroup of $\mathrm{SL}_{2}\left(p^{e}\right)$, so that $y^{e}$ fixes $p^{e}+4$ classes of $K$, and $p^{e}+3$ of $W$. But $y^{e}$ fixes the Steinberg character St of $K$, so by Brauer permutation lemma,

$$
f\left(\operatorname{Irr}\left(B_{0}\right), y^{e}\right)+f\left(\operatorname{Irr}\left(B_{1}\right), y^{e}\right)=f\left(\operatorname{Irr}\left(b_{0}\right), y^{e}\right)+f\left(\operatorname{Irr}\left(b_{1}\right), y^{e}\right) .
$$

Similarly, since $y^{e}$ fixes the two unipotent classes of $L / Z$, it follows by [7, Lemma 4.3] and the Brauer permutation lemma that $f\left(\operatorname{Irr}(K / Z) \backslash\{S t\}, y^{e}\right)$ $=f\left(\operatorname{Irr}(W / Z), y^{e}\right)$. But $L$ has only one block with maximal defect covering each linear character of $Z$, so

$$
f\left(\operatorname{Irr}\left(B_{i}\right), t\right)=f\left(\operatorname{Irr}\left(b_{i}\right), t\right)
$$

for $i=0,1$ and $t=y^{e}$. 
The values of $\chi_{(q \pm 1) / 2}^{(a)}$ on the classes $C^{(1)}$ and $C^{(2)}$ of $K$ (cf. [15, Lecture 5]) imply that $y^{e}$ fixes each character $\chi_{(q \pm 1) / 2}^{(a)}$ as $y^{e}$ fixes each $C^{(u)}$. Similarly, the values of $\psi_{(q-1) / 2}^{(a)}$ on the classes $C^{(3)}$ and $C^{(4)}$ of $W$ imply that $y^{e}$ fixes each character $\psi_{(q-1) / 2}^{(a)}$. Thus (7.5) holds when $d=m, r=2^{-1}$ and $t=y^{e}$, this and (7.7) imply that (7.5) holds for any $m, r$ and $t=y^{e}$. If $G / K$ is cyclic, then Proposition 7.6 follows by Lemma 7.5.

If $G / K$ is noncyclic, then we may suppose $x \in G$. Let $b_{i}^{\prime}(L)$ be the unique block of $L\langle x\rangle$ covering $b_{i}(L)$. Since the actions of $x$ and $y$ on $L$ are commutative, it follows that there is a bijection $\Phi: \operatorname{Irr}_{d}\left(B_{i}, \lambda,[r]\right) \rightarrow \operatorname{Irr}_{d}\left(b_{i}, \lambda,[r]\right)$ such that (7.6) holds for $t=x$ and $y$, and so for any $t \in \Gamma L_{2}(q)$ stabilizing $W$. A similar proof to that of Case (1) shows that the bijection $\Phi$ can be extended to a $\langle y\rangle$-set isomorphism of $\operatorname{Irr}_{d}\left(b_{i}^{\prime}(K), \lambda,[r]\right)$ and $\operatorname{Irr}_{d}\left(b_{i}^{\prime}(W), \lambda,[r]\right)$. Thus Proposition 7.6 follows by Lemma 7.5.

PROPOSITION 7.7. Let $G$ be a finite group, $B \in \operatorname{Blk}(G)$ with a defect group $D$, and $b$ the Brauer correspondent of $B$ in $N=N_{G}(D)$. Suppose that $B$ is any of the following:

(i) a block with cyclic, generalised quaternion or Klein-four defect group D such that $D / O_{p}(G)$ is $T I, O_{p}(G) \leqslant Z(G)$ and $O_{p}(G)$ is cyclic;

(ii) a 3-block of Suz, 2.Suz, 3.Suz, 6.Suz, Suz.2, 2.Suz.3, 3.Suz.2, $O^{\prime} N, 3 . O^{\prime} N$, $O^{\prime} N .2$.

Then Conjecture 1.3 holds for $B$.

Proof. Conjecture 1.3 holds when $D$ is cyclic by [20, Theorem (2.1)], and when $D$ is generalised quaternion or Klein-four by [31]. If $O_{p}(G) \leqslant Z(G)$ and $O_{p}(G)$ is cyclic, and $D / O_{p}(G)$ is cyclic or generalised quaternion, then Conjecture 1.3 holds by Corollary 6.2 (taking note of the remarks preceding that result). 3.

(ii) follows from [19] and [5] since the conjecture is identical to DPC for $p=$

\section{Acknowledgements}

We thank Burkhard Külshammer for his valuable suggestions. The second author also expresses his deep gratitude for the warm hospitality shown by the Department of Mathematics at the University of Auckland during his visit, when this research was done. We also thank the Marsden Fund for their support.

\section{References}

1. Alperin, J. L.: Local Representation Theory, University Press, Cambridge, 1986.

2. Jianbei An and Eaton, C. W.: The p-local rank of a block, J. Group Theory 3 (2000), 369-380.

3. Jianbei An and Eaton, C. W.: On TI and TI defect blocks, J. Algebra 243 (2001), 123-130.

4. Jianbei An and Eaton, C. W.: Blocks with trivial intersection defect groups, Math. Z. 247 (2004), 461-486. 
5. Jianbei An and O'Brien, E. A.: The Alperin and Dade conjectures for the O'Nan and Rudvalis simple groups, Comm. Algebra 30 (2002), 1305-1348.

6. Jianbei An and O'Brien, E. A.: Conjectures on the characters degrees of the Harada-Norton simple group HN, Israel J. Math. 137 (2003), 157-181.

7. Blau, H. I. and Michler, G. O.: Modular representation theory of finite groups with T. I. Sylow p-subgroups, Trans. Amer. Math. Soc. 319 (1990), 417-468.

8. Conway, J. H., Curtis, R. T., Norton, S. P., Parker, R. A. and Wilson, R. A.: Atlas of Finite Groups, Clarendon Press, Oxford, 1985.

9. Dade, E.: Counting characters in blocks II, J. Reine Angew. Math. 448 (1994), 97-190.

10. Dade, E.: Counting characters in blocks with cyclic defect groups, I, J. Algebra 186 (1996), 934-969.

11. Eaton, C. W.: On finite groups of $p$-local rank one and conjectures of Dade and Robinson, J. Algebra 238 (2001), 623-642.

12. Eaton, C. W.: The equivalence of some conjectures of Dade and Robinson, J. Algebra 271 (2004), 638-651.

13. Eaton, C. W. and Robinson, G. R.: On a minimal counterexample to Dade's projective conjecture, J. Algebra 249 (2002), 453-462.

14. Feit, W.: The Representation Theory of Finite Groups, North-Holland, Amsterdam, 1982.

15. Fulton, W. and Harris, J.: Representation Theory, Springer-Verlag, New York, 1991.

16. Geck, M.: Irreducible Brauer characters of the 3-dimensional special unitary groups in nondefining characteristic, Comm. Algebra 18(2) (1990), 563-584.

17. Gorenstein, D. and Lyons, R.: On finite groups of characteristic 2-type, Mem. Amer. Math. Soc. 276 (1982).

18. Harris, M. E. and Knörr, R.: Brauer correspondence for covering blocks of finite groups, Comm. Algebra 13(5) (1985), 1213-1218.

19. Himstedt, F.: Ann. of Math. (2) 156 (2002), 333-344.

20. Isaacs, I. M. and Navarro, G.: New refinements of the McKay conjecture for arbitrary finite groups, preprint.

21. Knörr, R. and Robinson, G. R.: Some remarks on a conjecture of Alperin, J. London Math. Soc. (2) 39 (1989), 48-60.

22. Külshammer, B., Okuyama, T. and Watanabe, A.: A lifting theorem with applications to blocks and source algebras, J. Algebra 232 (2000), 299-309.

23. Külshammer, B., Okuyama, T. and Watanabe, A.: On Laradji's character correspondence, preprint.

24. Külshammer, B. and Puig, L.: Extensions of nilpotent blocks, Invent. Math. 102 (1990), 17-71.

25. Laradji, A.: A character correspondence for twisted group algebras, J. Algebra 181 (1996), 926-933.

26. Puig, L., Pointed groups and construction of characters, Math. Z. 176 (1981), 265-292.

27. Robinson, G. R.: Local structure, vertices and Alperin's conjecture, Proc. London Math. Soc. (3) 72 (1996), 312-330.

28. Robinson, G. R.: Dade's projective conjecture for p-solvable groups, J. Algebra 229 (2000), 234-248.

29. Robinson, G. R.: Cancellation theorems related to conjectures of Alperin and Dade, J. Algebra 249 (2002), 196-219.

30. Suzuki, M.: Finite groups of even order in which Sylow 2-subgroups are independent, Ann. of Math. (2) 80 (1964), 58-77.

31. Uno, K.: Dade's conjecture for tame blocks, Osaka J. Math. 31 (1994), 747-772.

32. Uno, K.: Conjectures on character degrees for the simple Thompson groups, Osaka J. Math. 41 (2004), 11-36.

33. Watanabe, A.: On nilpotent blocks of finite groups, J. Algebra 163 (1994), 128-134. 